Revista Brasileira de Agricultura Irrigada v.13, nº.6, p. 3703 - 3713, 2019

ISSN 1982-7679 (On-line)

Fortaleza, CE, INOVAGRI - http://www.inovagri.org.br

DOI: $10.7127 /$ rbai.v13n6001134

Protocolo 1134.19 - 03/04/2020

Aprovado em 12/05/2020

\title{
SIMULAÇÃO MATEMÁTICA DO COMPORTAMENTO HIDRÁULICO DO GRADIENTE DE PRESSÃO EM LINHAS LATERAIS DE MICROIRRIGAÇÃO
}

\author{
Laís Barreto Franco ${ }^{1}$, Uriel Calisto Moura Pessoa ${ }^{2}$, Ceres Duarte Guedes Cabral de almeida ${ }^{3}$, João \\ Audifax Cezar de Albuquerque Filho ${ }^{4}$, Gustavo Barreto Franco ${ }^{5}$, Martiliana Mayani Freire ${ }^{6}$
}

\section{RESUMO}

A irrigação quando bem utilizada passa a ser um instrumento muito eficaz para o aumento da produtividade das culturas. Diante disso, o manejo da mesma consiste em aplicar a quantidade de água exata necessária à planta e no momento certo. Esta pesquisa objetivou simular por meio de modelos matemáticos, o comportamento hidráulico do gradiente de pressão de dois tipos de microaspersores fabricados pela Naandanjain, ao longo da linha lateral em nível, utilizando dados obtidos no catálogo do fabricante. A uniformidade de distribuição de água foi avaliada pelas equações propostas para uniformidade de emissão (EU) e uniformidade de emissão absoluta (EUa). Os resultados das simulações matemáticas para dois diferentes bocais da Naandanjain (black e orange) na linha lateral refletem um gradiente de pressão (Hi) e vazão (qi) com comportamento semelhante, logo, se a pressão do sistema for afetada por algum entupimento ou inclinação na lateral, consequentemente, a vazão também será afetada. A uniformidade de emissão, o coeficiente de variação de fabricação e a uniformidade de emissão absoluta para a pressão e vazão foram classificadas como excelente para ambos os bocais, e boa uniformidade de aplicação de água ao longo da linha lateral. Segundo o critério de uniformidade estatística também foi classificada como excelente, com coeficientes de pressão > 98\% e vazão > 99\% para ambos microaspesrsores estudados. Os resultados encontrados neste estudos sugerem que a utilização dos microaspersores, Naandanjain Black e Orange, é promissora, uma vez que, os coeficientes de uniformidade para vazão e pressão aproximaram-se de $100 \%$.

Palavras-chave: Irrigação localizada, coeficiente de uniformidade, vazão-pressão.

\footnotetext{
1 Mestre em Engenharia Agrícola, Universidade Federal Rural de Pernambuco, Recife, PE. E-mail: laisfranco.agro@yahoo.com.br

2 Mestrando em Engenharia Agrícola, Universidade Federal Rural de Pernambuco, Recife, PE; E-mail: uriel.pessoa2@gmail.com

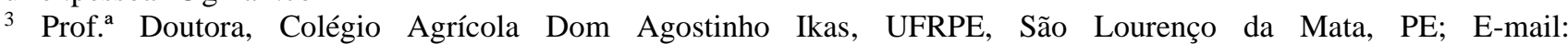
ceres.codai.ufrpe@gmail.com

${ }^{4}$ Prof. Doutor, Departamento de Engenharia Agrícola, UFRPE, Recife, PE. E-mail: jaudifaxfilho@yahoo.com.br

${ }^{5}$ Prof. Doutor, Departamento de Ciências Exatas e da Terra, UNEB, Salvador, BA. E-mail: gustavopraia@yahoo.com.br

${ }^{6}$ Doutoranda em Engenharia de Sistemas Agrícolas, ESALq/USP, Piracicaba, SP. E-mail: martilianafreire11@gmail.com
} 


\title{
MATHEMATICAL SIMULATION OF THE HYDRAULIC BEHAVIOR OF THE PRESSURE GRADIENT ON THE MICROIRRIGATION LATERAL LINES
}

\begin{abstract}
Irrigation when well used becomes a very effective instrument for increasing crop productivity. Thus, the irrigation management consists of the application of precise amount of water needed by the crop and at the right time. This research aimed to simulate by mathematics models the hydraulic behavior of the pressure gradient of two types of micro sprinklers manufactured by Naandanjain, along the lateral line based on data obtained from the manufacturer's brochure. The distribution uniformity was evaluated by the equations proposed for emission uniformity (EU) and absolute emission uniformity (EUa). The results of the mathematical simulation for two nozzles manufactured by Naandanjain (black and orange) on the lateral line reflect a pressure gradient (Hi) and flow (qi) with similar behavior, so if the system pressure is affected by some clogging or slope, the flow rate will also be affected. The emission uniformity, manufacturing variation coefficient and absolute emission uniformity for pressure and flow were classified as excellent for both nozzles, and good water application uniformity along the lateral line. According to statistical uniformity was also classified as excellent, with pressure coefficients $>98 \%$ and flow $>99 \%$ for both microsprinklers studied. These findings suggesting that the use of micro sprinklers, Naandanjain Black and Orange, is promising once the uniformity coefficients for flow and pressure were close to $100 \%$.
\end{abstract}

Keywords: Microirrigation, uniformity coefficient, pressure-flow.

\section{INTRODUÇÃO}

O aumento do consumo de água, devido ao crescimento populacional e atividades produtivas, contribuiu para escassez desse recurso natural em muitas regiões do mundo, que modificou hábitos, especialmente na agricultura irrigada. Portanto, a otimização dos sistemas de irrigação aliada ao planejamento da agricultura irrigada tornou-se indispensável, no sentido de compatibilizar os vários usos da água, monitorando a quantidade e a qualidade dos recursos hídricos (SILVA et al., 2016; PAZ et al., 2000).

Nesse contexto, a irrigação localizada é o método que permite aplicar água diretamente ao solo e próximo ao sistema radicular, em alta frequência e pequena intensidade, contribuindo para a economia de água, o que resulta em maior eficiência de aplicação (SANTANA et al., 2004).

Nesse sentido, o dimensionamento dos sistemas de irrigação devem possuir elevado grau de precisão, principalmente em áreas de topografia acidentada, em razão da acentuada variação de pressão no sistema, causando significativa diferença de vazão e, consequentemente, ocorre a redução na uniformidade de distribuição de água, na eficiência de aplicação da lâmina de água e aumento do escoamento superficial (LIMA et al., 2003). Assim, a verificação de parâmetros como, vazão dos emissores e volume de água fornecido durante o ciclo da cultura, possuem importância na agricultura irrigada, sobretudo, quando relacionados à uniformidade de distribuição de água (LEVIEN e FIGUEIRÊDO, 2013).

A microaspersão é um sistema intermediário entre a aspersão convencional e o gotejamento, a fim de aumentar a área molhada por emissor com menor custo fixo (FRIZZONE et al., 2012), funcionando com vazões de emissores variando de 20 a $150 \mathrm{~L} \mathrm{~h}^{-1}$, sendo maiores do que as dos gotejadores 1 a $20 \mathrm{~L} \mathrm{~h}^{-1}$ (DANTAS et al., 1997). Este sistema foi desenvolvido com o intuito de assegurar o suprimento de água em quantidades e períodos adequados, com uniformidade de distribuição aceitável em toda a área irrigada.

A microaspersão é, portanto, caracterizada por parâmetros hidráulicos como pressão de serviço e vazão dos emissores (SOUZA e BOTREL, 2004; FRIZZONE et al., 


\section{SIMULAÇÃO MATEMÁTICA DO COMPORTAMENTO HIDRÁULICO DO GRADIENTE DE PRESSÃO EM LINHAS LATERAIS DE MICROIRRIGAÇÃO}

2012; SILVA et al., 2012; NASCIMENTO et al., 2016).

A má distribuição de água na área é atribuída a falta de manutenção, sistemas mal dimensionados, entupimentos e/ou o fim da vida útil dos gotejadores ou microaspersores. Neste caso, poderá ocorrer uma fração de área irrigada com excesso e outras com déficit de água, não atendendo as necessidades hídricas das plantas. Portanto, uma das premissas do manejo racional da irrigação é manter o bom funcionamento dos equipamentos como também manter a pressão de serviço dentro dos limites especificados pelo fabricante (SILVA e SILVA, 2005). Prado et al. (2013) avaliaram as características hidráulicas e de distribuição de água de um canhão hidráulico, combinados com diferentes diâmetros de bocais e pressões de serviço. As variações no raio de alcance foram mais pronunciadas em condições de baixas pressões de serviço e para ângulos de jato menores, independente do bocal do aspersor. Ao submeter o sistema à pressão de serviço superior a $392 \mathrm{kPa}$, geraram maiores volumes de água aplicada a uma distância compreendida entre 60 e $80 \%$ do raio de alcance do aspersor. De forma geral, a pressão de serviço influencia diretamente o raio de alcance do jato (MARTINS et al., 2015; BORTOLUZZI e PRADO, 2017).De acordo com Botrel (1984), a relação entre vazão e pressão na entrada do microaspersor, a perda de carga localizada na inserção da linha lateral, o raio de alcance do jato, o diâmetro e a forma dos bocais constituem as principais características hidráulicas do microaspersor. Sendo assim, a utilização de equações explícitas que dimensionam hidraulicamente os sistemas de irrigação têm sido estudados com a finalidade de simplificar os cálculos e comparar metodologias que sejam mais adequadas para condições de ensaios hidráulicos (SOUSA e DANTAS NETO, 2014). O dimensionamento de linhas laterais de microaspersão deve seguir critérios que possibilitem alta uniformidade de distribuição de água. Para os emissores não compensados de pressão, a uniformidade de vazão ao longo da linha lateral depende da variação de pressão decorrente da perda de carga na tubulação (CARDOSO e FRIZZONE, 2007). Usualmente, os diâmetros nominais das tubulações de polietileno, para dimensionamento de linhas laterais são de 13 , 16, 20, 26, 32 ou $40 \mathrm{~mm}$, considerados pequenos diâmetros (GOMES, 1999; SOUSA e DANTAS NETO, 2014).

Diante do exposto, objetivou-se simular por meio de modelos matemáticos $\mathrm{o}$ dimensionamento de linhas laterais instaladas em nível com microaspersores, como alternativa para o conhecimento do desempenho de distribuição de pressão ao longo da mesma e seu efeito na umiformidade de emissão.

\section{MATERIAL E MÉTODOS}

$\mathrm{O}$ gradiente de energia para avaliar o desempenho da linha lateral, neste estudo, foi dimensionado a partir de simulações matemáticas para os emissores fabricados pela Naandanjain, bocais Black com diâmetro de $0,64 \mathrm{~mm}$ e vazão média de $24,5 \mathrm{~L} \mathrm{~h}^{-1}$, e Orange, com diâmetro de $1,50 \mathrm{~mm}$ e vazão de $33,2 \mathrm{~L} \mathrm{~h}^{-}$ 1. O conjunto dos dados iniciais para o desenvolvimento do trabalho foi oriundo do catálogo técnico fornecido pelo fabricante dos microaspersores (Tabela 1).

A linha lateral simulada tem como características ser de polietileno, com diâmetro nominal de $13 \mathrm{~mm}$, em condição de tubo liso, com comprimento máximo ajustado para pressão inicial 7 e $10 \mathrm{mca}$, até pressões máximas de 30 e 35 mca para os bocais Black e Orange, respectivamente. $\mathrm{O}$ critério de uniformidade considerado foi de $10 \%$ para variação de pressão ao longo da linha lateral. 
Tabela 1. Dados utilizados para simular a distribuição de energia na linha lateral

\begin{tabular}{lccccccc}
\hline Bocais & $\mathrm{PS}^{1}(\mathrm{mca})$ & $\mathrm{N}^{2}$ & $\mathrm{~L}^{3}(\mathrm{~m})$ & $\mathrm{E}^{4}(\mathrm{~m})$ & $\mathrm{T}^{5}\left({ }^{\circ} \mathrm{C}\right)$ & $\mathrm{m}^{6}$ & $\mathrm{D}^{7}(\mathrm{~m})$ \\
\hline Black & \multirow{2}{*}{20} & 20 & 66 & 3,3 & 20 & 1,7 & \multirow{2}{*}{0,013} \\
Orange & 19 & 62,7 & & & 5 & \\
\hline
\end{tabular}

${ }^{1}$ Pressão de serviço; ${ }^{2}$ Número de microaspersores; ${ }^{3}$ Comprimento da linha; ${ }^{4}$ Espaçamento entre emissores; ${ }^{5}$ Temperatura da água $;{ }^{6}$ Expoente da equação de perda de carga; ${ }^{7}$ Diâmetro interno da linha lateral.

Para processamento dos dados, desenvolveu-se um programa em linguagem MATLAB $^{\circledR}$, (versão R2007b licença 347698 Classroom UFRPE), que fornece o comprimento máximo da linha lateral, com base nas equações propostas por Denículi e Ramos (1992), em função da perda de carga admissível. O dimensionamento da linha lateral foi baseado em critérios de uniformidade, da perda de carga contínua, do fator de correção de perda de carga apresentado por Wu e Gitlin (1975), da vazão nominal do emissor operando na pressão de serviço de $20 \mathrm{mca}$, do espaçamento, das características hidráulicas do emissor e do diâmetro interno da tubulação de polietileno. Denículi e Ramos (1992) salientam que as perdas de carga acidentais podem ser negligenciadas, em face de seu pequeno valor diante da perda de carga contínua.

A modelagem do programa é alicerçada em bases teóricas apresentados por Detomini e Frizzone (2008). Assim, para o cálculo do comprimento da linha lateral (L) foram utilizados o número de aspersores $(\mathrm{N})$ e o espaçamento entre os emissores (Se). Posteriormente, para se obter a perda de carga admissível na linha lateral de múltiplas saídas (hf') (equação 1), foram calculadas a vazão total da linha lateral $(\mathrm{Q}), \mathrm{em} \mathrm{m}^{3} \mathrm{~s}^{-1}$ (equação 2), com base nos valores de descarga nominal do emissor (q); diâmetro interno da linha lateral (Di); a viscosidade cinemática da água (v), em $\mathrm{m}^{2} \mathrm{~s}^{-1}$, sendo considerada $1,01 \times 10^{-6} \mathrm{~m}^{2} \mathrm{~s}^{-1}$ para temperatura a $20^{\circ} \mathrm{C}$; fator de correção modificado $(\mathrm{F})$, com base na constante $\mathrm{m}$ de Blasius (equação 3); e, a perda de carga na linha lateral (hf) (equação 4), com base nos valores da pressão de serviço, variação de pressão com a pressão média nominal $(\mathrm{dH} / \mathrm{H})$ e expoente de fluxo do emissor (x). A equação hf' foi formulada pela combinação da equação de perda de carga de Darcy-Weisbach, com a equação de Blasius, para tubos lisos, e as adaptações para os distintos coeficientes de Blasius (C) e (m) propostos por Sousa e Dantas Neto (2014).

$$
\begin{aligned}
\mathrm{hf}^{\prime}=\frac{8 \cdot \mathrm{C}}{\pi^{(2-\mathrm{m})} \cdot 9,81} \cdot\left(\frac{4}{v}\right)^{(-\mathrm{m})} \cdot\left(\frac{\mathrm{q}}{\mathrm{Se}}\right)^{(2-\mathrm{m})} \cdot \mathrm{D}_{\mathrm{i}}^{-(5-\mathrm{m})} \cdot \mathrm{L}^{(3-\mathrm{m})} \cdot \mathrm{F} \\
\mathrm{Q}=\frac{\mathrm{q} \cdot \mathrm{L}}{\mathrm{Se}} \\
\mathrm{F}=\frac{1}{3-\mathrm{m}}
\end{aligned}
$$

Em que: $\mathrm{h}_{\mathrm{f}}$ - - perda de carga admissível na linha lateral de múltiplas saídas (mca); C coeficiente de atrito tabelado (adimensional); $\mathrm{m}$ - coeficiente de Blasius (adimensional); $v$ viscosidade cinemática da água $\left(\mathrm{m}^{2} \mathrm{~s}^{-1}\right) ; \mathrm{q}$ vazão nominal do emissor $\left(\mathrm{m}^{3} \mathrm{~s}^{-1}\right)$; $\mathrm{Se}$ - espaçamento entre os emissores (m); $\mathrm{Di}$ - diâmetro interno da linha lateral (mm); $\mathrm{L}-$ comprimento da linha lateral (m); F - fator de correção (adimensional); Q - vazão total da linha lateral $\left(\mathrm{m}^{3} \mathrm{~s}^{-1}\right)$.

$$
\mathrm{hf}=\mathrm{dH}=\frac{\mathrm{dq}}{\mathrm{q}} \frac{\mathrm{PS}}{\mathrm{x}}
$$


Em que:

hf - perda de carga na linha lateral (mca); $\mathrm{dH}$ variação de pressão nos extremos da linha lateral, ou seja, a diferença entre a pressão máxima e a mínima (mca); dq - variação de vazão $\left(\mathrm{L} \mathrm{h}^{-1}\right)$; q - vazão nominal $\left(\mathrm{L} \mathrm{h}^{-1}\right)$; PS pressão de serviço (mca); $\mathrm{x}$ - expoente de descarga do emissor.

Em seguida, foi calculado o comprimento relativo do ponto de inserção de cada emissor em relação ao início da lateral (CRi), por meio da equação 5 , com base nos valores de $\mathrm{L}$ e Se.

A simulação da variação de energia de energia de cada microaspersor foi realizada através do cálculo da pressão no início da linha lateral (Hi), pela equação 6, a partir da substituição do valor de CRi na equação proposta por $\mathrm{Wu}$ e Gitlin (1975).

Por conseguinte, calculou-se o vetor (qi), que representa a linha de distribuição de vazão dos emissores ao longo da tubulação lateral, sendo estimado pela equação vazão-pressão dos microaspersores selecionados para este estudo, equação 7.

$$
\begin{aligned}
& \mathrm{H}_{\mathrm{i}}=\mathrm{H}-\left[1-\left(1-\mathrm{CR}_{\mathrm{i}}\right)^{\mathrm{m}+1}\right] . \mathrm{hf}^{\prime} \\
& \mathrm{q}_{\mathrm{i}}=\mathrm{K} \cdot \mathrm{H}_{\mathrm{i}}^{\mathrm{x}}
\end{aligned}
$$

Em que: CRi - comprimento relativo do ponto de inserção de cada emissor em relação ao início da lateral $(\mathrm{m})$; i - posição do microaspersor; Hi - Pressão no início da linha lateral (mca); H - pressão máxima na entrada da lateral (mca); $\mathrm{q}_{\mathrm{i}}$ - vazão $\left(\mathrm{L} \mathrm{h}^{-1}\right) ; \mathrm{K}$ - coeficiente de descarga da equação vazão-pressão do emissor; $\mathrm{x}$ - expoente de descarga que caracteriza o regime de fluxo do emissor. Para os microaspersores selecionados, neste estudo, o regime de fluxo é classificado como turbulento, com expoente de descarga 0,5324 e 0,4495 para os bocais Black e Orange, respectivamente.
A avaliação das diferenças individuais entre os microaspersores ocorridas durante o processo de fabricação foi realizada por meio da determinação do coeficiente de variação de fabricação (CVf), equação 8 , e classificados (Tabela 2) segundo os critérios propostos por Solomon (1979).

$$
\mathrm{CVf}=\frac{\mathrm{Sq}}{\overline{\mathrm{q}}}
$$

Em que: CVf - coeficiente de variação de fabricação (\%); Sq - desvio padrão da vazão do emissor; $\overline{\mathrm{q}}$ - vazão média dos emissores $\left(\mathrm{L} \mathrm{h}^{-1}\right)$.

Tabela 2. Critérios para classificação do coeficiente de variação do fabricante (CVf)

\begin{tabular}{cc}
\hline $\operatorname{CVf}(\%)$ & Classificação \\
\hline$\leq 3,0$ & Excelente \\
$4,0-7,0$ & Média \\
$8,0-10,0$ & Marginal \\
$11,0-14,0$ & Pobre \\
$>15$ & Inaceitável \\
\hline
\end{tabular}

Fonte: Solomon (1979)

A uniformidade de distribuição de água ao longo da linha lateral foi avaliada por meio da determinação e classificação (Tabelas 3 e 4) dos coeficientes de uniformidade de emissão
(EU) (equação 9); uniformidade de emissão absoluta (EUa) (equação 10); e uniformidade estatística $\left(\mathrm{U}_{\mathrm{s}}\right)$ (equação 11) proposta por Bralts (1986). 


$$
\begin{aligned}
& E U=100\left(1-1,27 e^{-0,5} \mathrm{CVf}\right) \frac{\mathrm{q}_{n}}{\bar{q}} \\
& \mathrm{EU}_{\mathrm{a}}=50\left(\frac{\mathrm{q}_{\mathrm{n}}}{\overline{\mathrm{q}}}+\frac{\overline{\mathrm{q}}}{\mathrm{q}_{\mathrm{x}}}\right) \\
& U_{\mathrm{s}}=100\left(1-\mathrm{CV}_{\mathrm{q}}\right)=100\left(1-\frac{\mathrm{s}_{\mathrm{q}}}{\overline{\mathrm{q}}}\right)
\end{aligned}
$$

Em que: EU - uniformidade de emissão (\%); e - número de emissores por planta; $\mathrm{q}_{\mathrm{n}}$ - média das $25 \%$ menores vazões observadas $\left(\mathrm{L} \mathrm{h}^{-1}\right)$; $\mathrm{EU}_{\mathrm{a}}$ - uniformidade de emissão absoluta $(\%) ; \mathrm{q}_{\mathrm{x}}$
- média das 12,5\% maiores vazões observadas $\left(\mathrm{L} \mathrm{h}^{-1}\right) ; \mathrm{U}_{\mathrm{s}}-$ uniformidade estatística $(\%) ; \mathrm{CVq}$ - coeficiente de variação da vazão do emissor $(\%)$.

Tabela 3. Critérios para classificação da EU e EUa.

\begin{tabular}{cc}
\hline EU e EUa $(\%)$ & Classificação \\
\hline$\geq 90$ & Excelente \\
$80-90$ & Bom \\
$70-80$ & Regular \\
$<70$ & Ruim \\
\hline
\end{tabular}

Fonte: Favetta e Botrel (2001)

Tabela 4. Critérios para classificação da $U_{s}$.

\begin{tabular}{cc}
\hline $\mathrm{U}_{\mathrm{s}}(\%)$ & Classificação \\
\hline 290 & Excelente \\
$80-90$ & Muito bom \\
$70-80$ & Regular \\
$70-60$ & Péssimo \\
$<60$ & Inaceitável
\end{tabular}

Fonte: Favetta e Botrel (2001)

\section{RESULTADOS E DISCUSSÃO}

As simulações hidráulicas realizadas em microaspersores comerciais, por meio do método matemático proposto por Wu e Gitlin (1975), demonstraram bom desempenho do gradiente de pressão, ao dimensionar a linha lateral, fornecendo dados que podem ser utilizados como ferramenta de tomada de decisão em campo. O ajuste das equações potenciais de vazão, em função da pressão de serviço e do diâmetro do bocal (Figuras 1A e 1B) apresenta boa relação funcional entre os valores de vazão e de pressão, o qual pode ser observado pelos altos valores dos coeficientes de determinação $\left(\mathrm{R}^{2}=0,9972\right.$, para o bocal Black e $R^{2}=0,9907$, para o bocal Orange). Os microaspersores Naandanjain bocais Black e Orange apresentaram expoentes, para a variável pressão, nos valores de 0,5324 e 0,44495, respectivamente, classificando-os como emissores de regime turbulento, visto que o regime de escoamento dentro do emissor, representado pelo expoente $\mathrm{x}$, pode variar de 0 (autocompensado) a 1 (regime laminar), com valores de $\mathrm{x}=0,5$ para regime turbulento, indicando a suscetibilidade deste à variação de pressão de operação (CABELLO, 1996). 

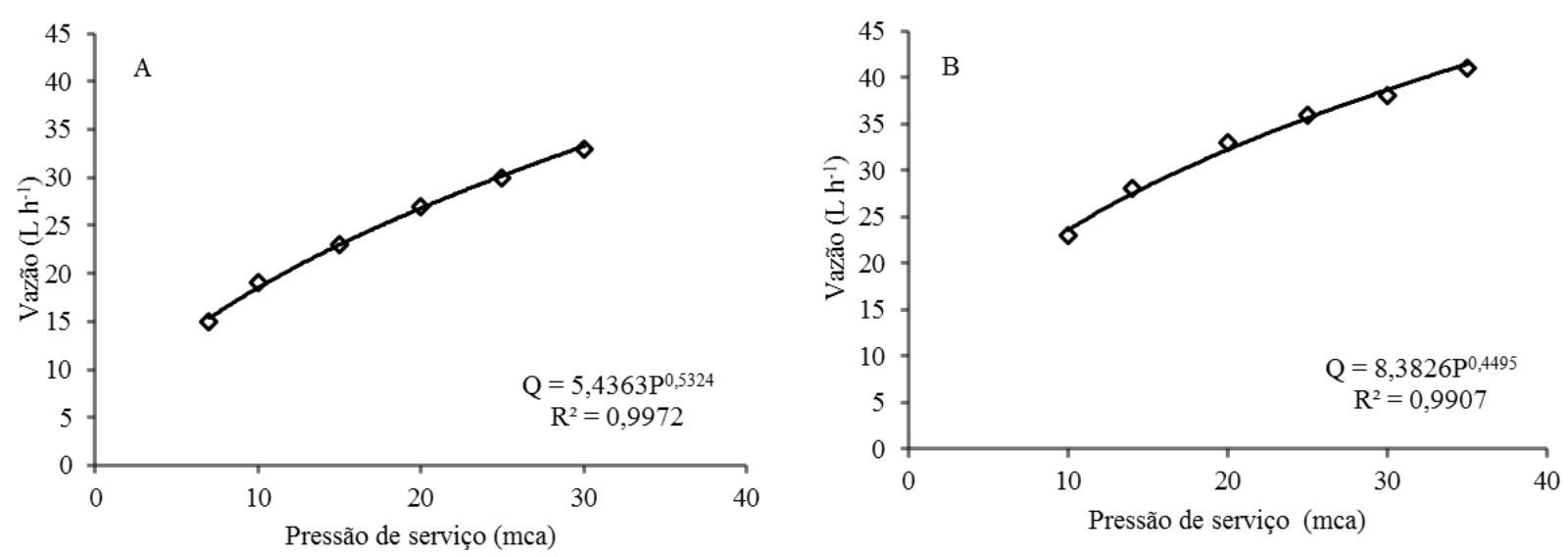

Figura 1. Vazão dos microaspersores em função da pressão de serviço para diferentes bocais. (A) Microaspersor Naandanjain Bocal Black; (B) Microaspersor Naandanjain Bocal Orange.

Em estudo conduzido por Bortoluzzi e Prado (2017), com o objetivo de modelar a distribuição de água do microaspersor Naan Hadar@, semelhante ao presente estudo, o valor encontrado do expoente de vazão foi próximo a 0,5 e na equação ajustada, o alto coeficiente de correlação $\left(\mathrm{R}^{2}=0,9948\right)$ aponta adequação dos dados ao modelo potencial. Ao analisar a relação existente entre a vazão, pressão e diâmetro estudados, observou-se que as perdas de carga foram proporcionais às potências utilizadas de 0,48 e 0,64; e que houve redução do coeficiente $\mathrm{f}$, obtido por Blasius, à medida em que se aumentou o diâmetro dos tubos de polietileno, conforme relataram FRIZZONE et al. (1998) e VILELA et al. (2003). Zitterell et al. (2009) ao estudarem a perda de carga em microtubos e conectores utilizados em microaspersão, relataram que as perdas de carga em função da vazão e diâmetro estudados apresentaram ajuste com $\mathrm{R}^{2}$ superior a 0,9990 para o modelo potencial avaliado e redução do coeficiente $\mathrm{f}$, determinado por Blasius, quando maior foi o diâmetro dos tubos.

Os valores de coeficiente de variação de fabricação (CVf) em função da pressão dos microaspersores de regime turbulento foram inferiores a 2,5 e 2,2\% para os bocais Black e Orange, respectivamente. Estes resultados caracterizam os microaspersores como excelentes, conforme descrito por Solomon (1979), quanto à uniformidade, material utilizado e processo de fabricação, demonstrando alta qualidade dos bocais utilizados no presente estudo e pequena variação na vazão ocasionada pelo processo de fabricação. Resultados semelhantes foram obtidos por Holanda Filho et al. (2001), ao realizarem a caracterização hidráulica do microaspersor Rondo, obtiveram CVf médio de $2,8 \%$, sendo, portanto, classificado como excelente.

Em estudo conduzido por Pinheiro et al. (2019) com o intuito de simular o comportamento teórico-hidráulico de microaspersor do mesmo fabricante deste estudo, bocal verde, diâmetro de $1,0 \mathrm{~mm}$ e vazão média de $51,12 \mathrm{~L} \mathrm{~h}^{-1}$, os autores constataram que o modelo matemático apresentou excelente ajuste do comportamento potencial da vazão em relação a pressão, com $\mathrm{R}^{2}=0,98$. A relação vazão-pressão apresentou excelente uniformidade de distribuição ao longo da linha lateral, com EU para a pressão e vazão de 94,42 e 97,90 \%, respectivamente.

Os valores encontrados para uniformidade de emissão (EU) e uniformidade de emissão absoluta $\left(E U_{a}\right)$, para o bocal Black, foram $94,69 \%$ e $96,68 \%$, respectivamente. E, para o bocal Orange, $97,72 \%$ para EU e $96,37 \%$ para $\mathrm{EU}_{\mathrm{a}}$, sendo classificados segundo Bralts (1986) como excelentes. Estes resultados apontam uma boa uniformidade de distribuição e aplicação de água ao longo da linha lateral, o que influencia no rendimento das culturas, visto que a uniformidade no sistema de irrigação acarreta na aplicação do volume adequado de água e redução de perdas de nutrientes por lixiviação (VALNIR JUNIOR et al., 2016). 
Ao longo da linha lateral foi verificada variação de pressão para o bocal Black de 19,08 a 22,08 mca (Figura 2A) e para o Orange de 18,86 a 22,54 mca (Figura 2B). Os microaspersores Naandanjain bocais Black e Orange apresentaram excelente uniformidade na distribuição de água ao longo da linha lateral com uniformidade estatística de 99,91\% e $99,30 \%$, respectivamente, sendo classificados como excelentes conforme Bralts (1986). Em relação à uniformidade da pressão, os valores foram de $99,76 \%$ e $98,84 \%$, respectivamente. Segundo Silva e Silva (2005), a uniformidade da aplicação da lâmina de irrigação ao longo da linha lateral, em sistemas de irrigação localizada, está diretamente relacionada com a variação da pressão e vazão dos emissores, desta forma, alterações na pressão ocasionadas pela perda de carga ocasionará variação na vazão.

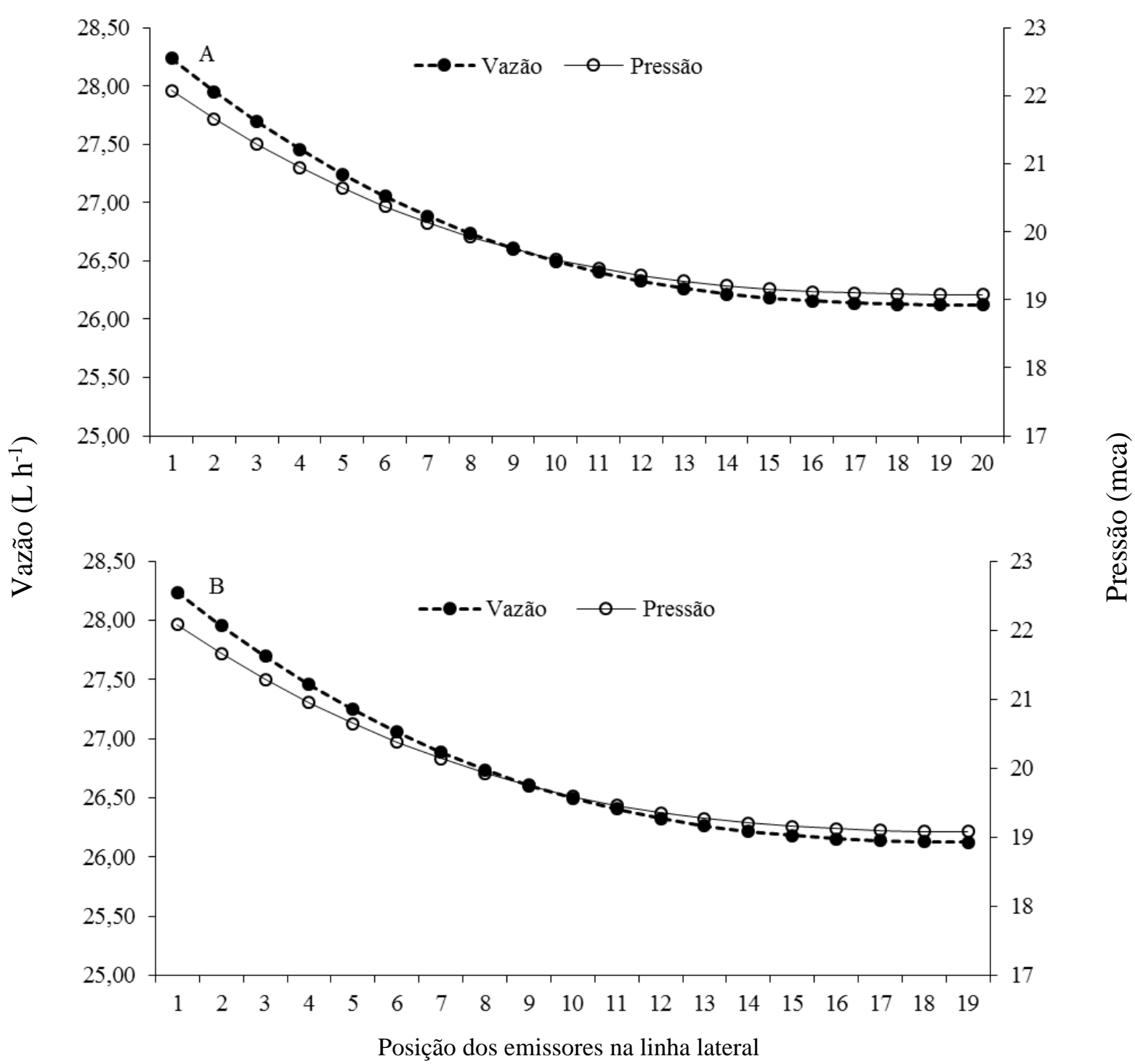

Figura 2. Variação da pressão e vazão na linha lateral em função da posição dos microaspersores. (A) Microaspersor Naandanjain Bocal Black; (B) Microaspersor Naandanjain Bocal Orange.

Resultado semelhante com microaspersor do mesmo fabricante deste estudo foi encontrado por Batista et al. (2017), quando simularam o comportamento hidráulico do microaspersor de bocal violeta, diâmetro de $0,82 \mathrm{~mm}$ e vazão média de $39 \mathrm{~L} \mathrm{~h}^{-1}$ para uma pressão de serviço de 25 mca. A relação vazãopressão decresceu ao longo da linha lateral, apesar de haver excelente uniformidade na distribuição de água ao longo da mesma, com coeficiente de uniformidade para a pressão e vazão de 94,42 e 75,22 \%, respectivamente. 
Resultados próximos, também foram obtidos, por Bortoluzzi e Prado (2017), estudando a uniformidade de distribuição de água do microaspersor Naan 7110, fazendo uso de quatro diâmetros de bocais $(0,9 ; 1,0 ; 1,1$ e 1,2 $\mathrm{mm}$ ), obtendo uniformidade de vazão próxima a $80 \%$ para pressão de serviço de 15 mca. Ainda segundo os referidos autores, o resultado positivo da uniformidade da vazão está associado a pressão de serviço como também a área efetivamente irrigada, em torno de 80 e $90 \%$ do raio de projeção. Para Silva e Silva (2005), a pressão de serviço deve funcionar dentro dos limites de pressão especificados pelo fabricante, para não ocorrer diminuição do raio de alcance do microaspersor.

\section{CONCLUSÕES}

O algoritmo desenvolvido neste estudo foi capaz de fornecer o comprimento máximo ajustado da linha lateral, o número de emissores para tubulação lateral de polietileno e avaliar a performance dos sistemas de microirrigação, podendo ampliar as alternativas de projeto hidráulico de sistemas de irrigação localizada a partir de informações das características hidráulicas de vários emissores, gotejadores ou microaspersores, e subsidiar na decisão sobre o modelo do emissor.

Os microaspersores Naandanjain Black e Orange apresentam classificação excelente, ao considerar os coeficientes de variação de fabricação, a uniformidade de emissão e uniformidade de emissão absoluta, demonstrando pequena variação na vazão devido ao processo de fabricação e boa uniformidade de aplicação de água no sistema de irrigação. Os microaspersores, Naandanjain Black e Orange, apresentaram excelente uniformidade estatística ao longo da linda lateral em nível, com coeficientes de pressão de $99,76 \%$ e $98,84 \%$, e vazão $99,91 \%$ e $99,30 \%$, respectivamente. Os resultados são promissores para os microaspersores estudados, uma vez que, os coeficientes de uniformidade para vazão e pressão aproximaram-se de $100 \%$.

\section{REFERÊNCIAS BIBLIOGRÁFICAS}

BATISTA, P. H. D.; BARROS, B. E. A.; SILVA, J. L. B. da.; PINHEIRO, A. B.; COUTINHO, A. S. Simulação do comportamento hidráulico de microaspersores e microtubos ao longo da linha lateral. Tecnologia \& Ciência Agropecuária, v. 11, n. 1, p. 67-71, 2017.

BORTOLUZZI, D. D. O.; PRADO, G. Modelagem da distribuição de água de microaspersores. Revista Brasileira de Agricultura Irrigada, v. 11, n. 7, p. 20632075, 2017. DOI: 10.7127/rbai.v11n700683

BOTREL, T.A. Hidráulica de microaspersores e linhas laterais para irrigação localizada. 1984. 78 f. Dissertação ESALQ/USP, Piracicaba.

BRALTS, V. F. Field performance and evaluation. In: Developments in Agricultural Engineering. Elsevier, v. 9, n. 1, p. 216-240, 1986.

CABELLO, F. P. Riegos localizados de alta frecuencia (RLAF): goteo, microaspersión, exudación. 3.ed. Madrid: Mundi-Prensa, 1996. $513 \mathrm{p}$.

CARDOSO, G.G.G.; FRIZZONE, J.A. Perda de carga em linhas laterais de microirrigação. BloEngenharia, v. 1, n. 2, p. 147-162, 2007. DOI: $10.18011 /$ bioeng2007v1n2p147-162

DANTAS, J.N.; MEDEIROS, M. G. A.; AZEVEDO, C.A.V.; AZEVEDO, H.M. Performance hidráulica e perfil de distribuição de água do microaspersor na NA 7110, sob diferentes condições de ventos. Revista Brasileira de Engenharia Agrícola e Ambiental, v. 1, n. 1, p. 57-61, 1997.

DENICULI, W.; RAMOS, M. M. Perda de carga continua em tubulações dotadas de múltiplas saídas. Revista Ceres, v. 39, n. 222, p. 129-152, 1992. 
DETOMINI, E. R.; FRIZZONE, J. A. Bases teóricas e fundamentos para otimização hidráulica do comprimento de linhas laterais em microirrigação. Revista Brasileira Agricultura Irrigada, v. 2, n. 1, p. 16-23, 2008. DOI: 10.7127/RBAI.V2N100007

FAVETTA, G.M.; BOTREL, T.A. Uniformidade de sistemas de irrigação localizada: validação de equações. Scientia Agricola, v. 58, n. 2, p. 427-430, 2001. DOI: 10.1590/S0103-90162001000200030

FRIZZONE, J. A.; VIEIRA, A. T.; PAZ, V. P. S. Caracterização hidráulica de um tubo gotejador. Revista Brasileira de Engenharia Agrícola e Ambiental, Campina grande, v. 2, n. 3, p. 278-283, 1998.

FRIZZONE, J. A; LOURENÇO, P. S.; REZENDE, R.; FARIA, M. A. Microirrigação, gotejamento e microaspersão. Maringá:Editora EDUEM, 2012. 365 p.

GOMES, H. P. Engenharia de irrigação: hidráulica dos sistemas pressurizados, aspersão e gotejamento. 3.ed. Campina Grande: Ed. UFPB, 1999. 412p.

HOLANDA FILHO, R. S. F.; PORTO FILHO, F. Q.; MIRANDA, N. O.; MEDEIROS, J. F. Caracterização hidráulica do microaspersor Rondo, da plastro. Revista Brasileira de Engenharia Agrícola e Ambiental, v. 5, n. 1, p. 16-21, 2001. DOI: 10.1590/S141543662001000100004

LEVIEN, S. L. A.; FIGUEIRÊDO, V. B. Metodologia simplificada para a estimativa em campo da uniformidade de sistemas de irrigação por gotejamento superficial. Revista Brasileira de Agricultura Irrigada, v. 7, n. 5, p. 290-299, 2013. DOI: 10.7127/RBAI.V7N500179

LIMA, S. C.; FRIZZONE, J. A.; COSTA, R. N.; SOUZA, F. D.; PEREIRA, A. S.; MACHADO, C. C.; VALNIR JÚNIOR, M. Curvas de desempenho de válvulas reguladoras de pressão novas e com diferentes tempos de utilização. Revista Brasileira de Engenharia Agrícola e Ambiental, v. 7, n. 2, p. 201-209, $2003 . \quad$ DOI: $\quad 10.1590 /$ S141543662003000200003

MARTINS, P. E. S.; SILVA, E. R. da.; SANTANA, V. G.; ARAÚJO, J. A. de.; ZANINI, N. J. Z. Uniformidade de distribuição de água do microaspersor MC-20. Revista de Ciências Agrárias, v. 38, n. 1, p. 65-70, 2015.

NASCIMENTO, R. C.; MEDEIROS, P. R. F.; SANTOS, G. S.; ALENCAR, E. C. P. V.; GONÇALVES, D. R.; MARTINS, A. P. F. Dinâmica da obstrução de emissores usados na cultura da uva utilizando água salina de poço subterraneo. Irriga, v. 21, n. 1, p. 491-502, 2016. DOI: $10.15809 /$ irriga.2016v21n3p491502

PAZ, V. P. S.; TEODORO, R. E. F.; MENDONCA, F. C. Recursos hídricos, agricultura irrigada e meio ambiente. Revista Brasileira de Engenharia Agrícola e Ambiental, v. 4, n. 3, p. 465-473, 2000. DOI: 10.1590/S1415-43662000000300025

PINHEIRO, A. G.; SILVA, J. L. B.; SILVA, D. A. O.; BATISTA, P. H. D.; RODRIGUES, J. A. M.; SILVA, M. V. Simulação matemática do comportamento teórico-hidráulico de microaspersores e microtubos ao longo da linha lateral. Revista Ambientale, v. 11, n. 2, p. 2233, 2019. DOI: 10.34032/ambientale.v11i2.95

PRADO, G. do.; FARIA, L C.; OLIVEIRA, H. F. E. de.; COLOMBO, A. Efeito do ângulo do jato nas características técnicas de um canhão hidráulico. Revista Brasileira de Engenharia Agrícola e Ambiental, v. 17, n. 7, p. 689-697, $2013 . \quad$ DOI: $\quad 10.1590 /$ S141543662013000700001

SANTANA, M. S.; OLIVEIRA, C. A. S.; QUADROS, M. Crescimento inicial de duas cultivares de cafeeiro adensado influenciado por níveis de irrigação localizada. Revista Engenharia Agrícola, v. 24, n. 3, p. 644-653, 
$2004 . \quad$ DOI:

69162004000300016

SILVA, C. A. da; SILVA, C. J. da. Avaliação de uniformidade em sistemas de irrigação localizada. Revista Científica Eletrônica de Agronomia, ano IV, n. 8, 2005.

SILVA, L. P.; SILVA, M. M.; CORREA, M. M.; SOUZA, F. C. D.; SILVA, E. F. F. E. Desempenho de gotejadores autocompensantes com diferentes efluentes de esgoto doméstico. Revista Brasileira de Engenharia Agrícola e Ambiental, v. 16, p. 480-486, 2012. DOI: 10.1590/S1415-43662012000500003

SILVA, P. F.; MATOS, R. M.; NETO, J. D.; GOMES, R. C. B.; LIMA, V. L. A. Characterization of the hydraulic performance of self-compensating emitters with water of different salt concentrations. Semina: Ciências Agrárias, v. 37, n. 2, p. 639-650, 2016. DOI: 10.5433/1679-0359.2016v37n2p639

SOLOMON, K. Manufacturing variation of trickle emitters. Transactions of the ASAE, St. Joseph, v. 22, n. 5, p. 1034-1038, 1979.

SOUSA, R. O. R. M.; DANTAS NETO, J. Equação explicita para cálculo do fator de atrito de Darcy-Weisbach em projetos de irrigação pressurizada. Irriga, v. 19, n. 1, p. 137- 148, 2014. DOI: 10.15809/irriga.2014v19n1p137

SOUZA, R. R. M.; BOTREL, T. T. Modelagem para o dimensionamento de microtubos em irrigação localizada. Revista Brasileira de Engenharia Agrícola e Ambiental, v. 8, n. 1, p. $1-7,2004$. DOI: $10.1590 / \mathrm{S} 1415-$ 43662004000100003

VALNIR JÚNIOR, M.; ROCHA, J. P. A.; DEMONTIÊZO, F. L. L.; LIMA, L. S. S.; CARVALHO, C. M.; CARVALHO, L. L. S. Análise comparativa de metodologia de coleta de dados para avaliação de sistemas de irrigação localizada. Revista Brasileira de Agricultura Irrigada, v. 10, n. 5, p. 965-975, 2016. DOI: $10.7127 /$ rbai.v10n500477

VILELA, L. A. A.; SOCCOL, O. J.; GERVÁZIO, E. S.; FRIZZONE, J. A.; BOTREL, T. A. Alteração no diâmetro de tubos de polietileno submetidos a diferentes pressões. Revista Brasileira de Engenharia Agrícola e Ambiental, Campina Grande, v.7, n. 1, p. 182-185, 2003. DOI: $10.1590 / \mathrm{S} 1415-$ 43662003000100030

WU, I. P.; GITLIN, H. M. Energy gradient line for drip irrigation laterals. Journal of the Irrigation and Drainage Division, v. 101, n. 4, p. 323-326, 1975.

ZITTERELL, D. B.; FRIZZONE, J. A.; RETTORE NETO, O.; ULLMANN, M. N. Perda de carga em microtubos e conectores utilizados em microaspersão. Engenharia Agrícola, v. 29, n. 4, p. $591-604$, $2009 . \quad$ DOI:10.1590/ S0100-69162009000400009 